\title{
Correction: Trinh, H.V., et al. Humoral Response to the HIV-1 Envelope V2 Region in a Thai Early Acute Infection Cohort. Cells 2019, 8, 365
}

\author{
Hung V. Trinh ${ }^{1,2, \dagger}$, Neelakshi Gohain ${ }^{1,2, \dagger}$, Peter T. Pham ${ }^{1,2}$, Christopher Hamlin ${ }^{1,2, \ddagger}$, \\ Hongshuo Song ${ }^{1,2}$, Eric Sanders-Buell ${ }^{1,2}$, Meera Bose ${ }^{1,2} ®$, Leigh A. Eller ${ }^{1,2}$, Swati Jain ${ }^{3}$, \\ Gherman Uritskiy ${ }^{3}{ }^{(D}$, Venigalla B. Rao ${ }^{3}$, Sodsai Tovanabutra ${ }^{1,2}$, Nelson L. Michael ${ }^{1}$ (i), \\ Merlin L. Robb ${ }^{1,2}$, M. Gordon Joyce ${ }^{1,2, * \mathbb{D}}$ and Mangala Rao ${ }^{1, *}$
}

1 U.S. Military HIV Research Program, Walter Reed Army Institute of Research, Silver Spring, MD 20910, USA; htrinh@hivresearch.org (H.V.T.); ngohain@hivresearch.org (N.G.); ppham@hivresearch.org (P.T.P.); christopher.hamlin@nih.gov (C.H.); hsong@hivresearch.org (H.S.); esandersbuell@hivresearch.org (E.S.-B.); mbose@hivresearch.org (M.B.); leller@hivresearch.org (L.A.E.); Stovanabutra@hivresearch.org (S.T.); nmichael@hivresearch.org (N.L.M.); mrobb@hivresearch.org (M.L.R.)

2 Henry M. Jackson Foundation for the Advancement of Military Medicine, Inc., Bethesda, MD 20817, USA

3 Department of Biology, The Catholic University of America, Washington, DC 20064, USA; 80jain@cua.edu (S.J.); guritsk1@jhu.edu (G.U.); rao@cua.edu (V.B.R.)

* Correspondence: gjoyce@hivresearch.org (M.G.J.); mrao@hivresearch.org (M.R.); Tel.: +1-(301)-319-7528 (M.G.J.); +1-(301)-319-7699 (M.R.)

+ These authors contributed equally to this work.

$\ddagger$ Current address: DAIDS, National Institute of Allergy and Infectious Diseases, Rockville, MD 20852, USA.

In the original version of our article [1], insufficient acknowledgement was given for initial analysis of RV217 participant 40007 HIV-1 viral sequences, and for identification of HIV-1 viral variants with His or Tyr at position 173 and a short deletion DSY in the V2 loop, likely due to host immune pressure that results in significant Env antigenic changes. We apologize for the original error. To correct this oversight, Swati Jain, Gherman Uritskiy, and Venigalla B. Rao have been added as authors, and the acknowledgements have been altered to appropriately recognize support and funding.

The corrected author list and author contributions are provided below:

Hung V. Trinh 1,2, Hongshuo Song 1,2, Eric Sanders-Buell 1,2, Meera Bose 1,2, Leigh A. Eller 1,2, Swati Jain ${ }^{3}$, Gherman Uritskiy $^{3}$, Venigalla B. Rao ${ }^{3}$, Sodsai Tovanabutra 1,2, Nelson L. Michael ${ }^{1}$, Merlin L. Robb ${ }^{1,2}$, M. Gordon Joyce ${ }^{1,2, *}$ and Mangala Rao ${ }^{1, *}$

1 U.S. Military HIV Research Program, Walter Reed Army Institute of Research, Silver Spring, MD 20910, USA; htrinh@hivresearch.org (H.V.T.); ngohain@hivresearch.org (N.G.); ppham@hivresearch.org (P.T.P.); christopher.hamlin@nih.gov (C.H.); hsong@hivresearch.org (H.S.); $\quad$ esandersbuell@hivresearch.org (E.S.-B.); mbose@hivresearch.org (M.B.); leller@hivresearch.org (L.A.E.); Stovanabutra@hivresearch.org (S.T.); nmichael@hivresearch.org (N.L.M.); mrobb@hivresearch.org (M.L.R.)

2 Henry M. Jackson Foundation for the Advancement of Military Medicine, Inc., Bethesda, MD 20817, USA

3 Department of Biology, The Catholic University of America, Washington, DC 20064, USA; 80jain@cua.edu (S.J.); guritsk1@jhu.edu (G.U.); rao@cua.edu (V.B.R.)

* Correspondence: gjoyce@hivresearch.org (M.G.J.); mrao@hivresearch.org (M.R.); Tel.: +1-(301)-319-7528 (M.G.J.); +1-(301)-319-7699 (M.R.) 
$+\quad$ These authors contributed equally to this work.

$\ddagger \quad$ Current address: DAIDS, National Institute of Allergy and Infectious Diseases, Rockville, MD 20852, USA.

Author Contributions: M.R., M.G.J., M.L.R., H.V.T., and N.G. conceived and designed the experimental approach, and analyzed and interpreted the results. H.V.T. and C.H. conducted the Biacore analysis; N.G. and M.G.J. performed structure-based modeling; P.T.P., H.S., and S.T. generated and analyzed viral sequences; E.S.-B. and M.B. performed SGA; S.J. and G.U. performed phylogenetic and immune escape analysis; V.B.R. conceived and oversaw the experiments and analysis conducted at CUA; L.A.E., M.L.R., and N.L.M. conducted the RV217 study and provided the samples. M.R. and M.G.J. oversaw the experiments and analysis. H.V.T., N.G., M.G.J., and M.R. wrote and finalized the manuscript. All authors read and approved the final manuscript.

Funding: This work was supported by the Department of Defense through cooperative agreements (W81XWH-18-2-0040) with the Henry M. Jackson Foundation for the Advancement of Military Medicine Inc. and the U.S. Department of Defense, and by the National Institute of Allergy and Infectious Diseases, NIH, through an interagency agreement with the U.S. Army (Y1-AI-2642-17). H.V.T. was partially supported by a grant from the Swiss National Science Foundation (P3SMP3_148406/1). V.B.R. was supported by a grant from NIAID, NIH (AI102725), and a subaward from the Henry M. Jackson Foundation (W81XWH-11-2-0174).

\section{Reference}

1. Trinh, H.V.; Gohain, N.; Pham, P.T.; Hamlin, C.; Song, H.; Sanders-Buell, E.; Bose, M.; Eller, L.A.; Tovanabutra, S.; Michael, N.L.; et al. Humoral Response to the HIV-1 Envelope V2 Region in a Thai Early Acute Infection Cohort. Cells 2019, 8, 365. [CrossRef] [PubMed]

(C) 2019 by the authors. Licensee MDPI, Basel, Switzerland. This article is an open access article distributed under the terms and conditions of the Creative Commons Attribution (CC BY) license (http://creativecommons.org/licenses/by/4.0/). 